FORMATION Formation emploi

Revue française de sciences sociales

100 | octobre-décembre 2007

De la formation professionnelle en Suisse

\title{
Les entreprises suisses : un investissement faible et sélectif dans la formation continue
}

Continuing vocational training at Swiss firms: a low and selective investment Schweizerische Unternehmen: nur wenige und selektive Investitionen für die

Weiterbildung

Siegfried Hanhart

\section{(2) OpenEdition}

Journals

Édition électronique

URL : http://journals.openedition.org/formationemploi/1270

DOI : 10.4000/formationemploi.1270

ISSN : 2107-0946

Éditeur

La Documentation française

Édition imprimée

Date de publication : 1 octobre 2007

Pagination : $65-78$

ISSN : 0759-6340

Référence électronique

Siegfried Hanhart, «Les entreprises suisses : un investissement faible et sélectif dans la formation continue », Formation emploi [En ligne], 100 | octobre-décembre 2007, mis en ligne le 01 octobre 2009 consulté le 30 octobre 2020. URL : http://journals.openedition.org/formationemploi/1270 ; DOI https://doi.org/10.4000/formationemploi.1270

(c) Tous droits réservés 


\section{DOSSIER}

\section{Les entreprises suisses: un investissement faible et sélectif dans la formation continue}

Par Siegfried Hanhart*

La reconnaissance par les pouvoirs publics du rôle que pourraient jouer les entreprises dans la qualification professionnelle des adultes devrait avoir pour corollaire que les firmes considèrent leurs dépenses de formation professionnelle continue comme de véritables investissements.

Dans cet article, nous nous intéressons à la formation professionnelle continue (FPC) en Suisse, et plus particulièrement aux activités soutenues par les entreprises. Précisons que dans ce pays, la FPC des salariés en activité repose largement sur l'initiative et l'engagement des employés et des employeurs. Dans ce contexte, il est essentiel de connaître le comportement des entreprises dans le maintien, voire le développement des compétences de leurs collaborateurs ${ }^{1}$. À cette fin, nous avons mené récemment une recherche sur les investissements en FPC des entreprises privées et publiques. Nous avons confronté quelques résultats helvétiques aux données européennes de l'enquête CVTS2 2 . Cette comparaison est

\footnotetext{
${ }^{1}$ Nous utilisons indifféremment les termes de collaborateurs, de salariés, d'employés pour désigner des hommes et des femmes.

${ }^{2}$ L'enquête CVTS2 a été menée, en 2000/2001, auprès de 76000 entreprises de 10 employés et plus dans les 15 pays alors membres de l'Union européenne, en Norvège et dans les 9 pays candidats. Elle portait sur le volume et le contenu de la formation professionnelle en entreprise, ainsi que sur les politiques et la gestion de la formation.
}

intéressante dans la mesure où l'économie suisse se caractérise par une forte compétitivité des entreprises d'exportation et par de faibles taux de chômage par rapport à ceux de la plupart des États membres de l'Union européenne ; cependant, en 2006, la Suisse a enregistré une faible croissance de son PIB (produit intérieur brut) en comparaison internationale. Nous concluons notre contribution sur l'apport d'une telle recherche à la définition d'une politique de formation continue en Suisse.

* Siegfried Hanhart est professeur adjoint en planification de l'éducation à la faculté de psychologie et des sciences de l'éducation de l'université de Genève. II a récemment publié : Hanhart S. (2005), " Les entreprises et l'apprentissage tout au long de la vie: un conflit de valeurs? ”, Revue Questions Vives, 6, pp. 123-131. Hanhart S. (2005), "L'évaluation des établissements d'enseignement tertiaire : contrainte ou opportunité ? », L'éducation en débats : analysée comparée, 3, pp. 52-62. 


\section{UN CONTEXTE POLITIQUE EN MUTATION}

Traditionnellement, en Suisse, la FPC relève de la responsabilité des individus et des entreprises. Au cours des vingt dernières années, on observe toutefois une évolution du rôle des pouvoirs publics, liée à la conjoncture économique. À la fin des années 80, l'économie helvétique manque de main-d'œuvre qualifiée et le Parlement fédéral adopte, pour la première fois, différentes mesures visant à stimuler la FPC. Quelques années plus tard, avec l'apparition d'une récession économique, les finalités assignées à la FPC par les autorités fédérales s'élargissent: la FPC doit désormais contribuer à la réinsertion sociale des chômeurs. Enfin, l'avènement de la société de l'information incite le Conseil fédéral à adopter, en 1998, une stratégie d'accès et d'utilisation des nouvelles technologies de la communication et de l'information; une attention particulière est accordée aux offres de formation pour les adultes (Schlaefli \& Gonon, 1999, pp. 63-65).

Nous serions tentés d'écrire que la conjoncture économique a, en quelque sorte, contraint les pouvoirs publics à intervenir dans le domaine de la FPC, sans pour autant bouleverser le paysage de cette dernière.

Qu'il s'agisse de maintenir ou de développer les compétences des salariés, de réinsérer des demandeurs d'emploi ou d'élaborer une politique publique de FPC, une collaboration entre pouvoirs publics et employeurs est nécessaire, particulièrement dans un pays où l'action de l'État est régie par un principe de subsidiarité. Or, jusqu'à présent, on disposait de peu d'informations sur le comportement des employeurs face à la FPC de leurs collaborateurs. Relevons encore qu'en Suisse les entreprises n'assument pas d'obligation légale en matière de FPC de leur personnel, à quelques rares exceptions (médecins, experts-comptables, ...).

Il existe différentes façons d'appréhender l'attitude des employeurs face à la FPC. Un programme de recherche intitulé « Formation et emploi », lancé par le Fonds national suisse de la recherche scientifique, nous a permis de mener une étude sur les dépenses de

\section{Encadré 1}

\section{La formation continue dans le contexte politique helvétique}

En matière de FPC (formation professionnelle continue), la Confédération (pouvoir central) et les cantons agissent selon un principe de subsidiarité, "c'est-à-dire que l'action de l'État se réalise en complémentarité avec celle des autres acteurs [...] là où le marché n'intervient pas» (OCDE, 2001 a, p. 21). Par ailleurs, en l'absence d'un article dans la Constitution fédérale sur la formation, la Confédération, jusqu'à aujourd'hui, s'est appuyée, dans ses interventions dans le domaine de la FPC, sur des dispositions relevant des lois fédérales sur la formation professionnelle et sur l'assurance-chômage, voire sur des arrêtés fédéraux à durée limitée. Les cantons peuvent mettre en œuvre leurs propres mesures d'encouragement de la FPC, dès lors qu'ils en assument le financement. Cette situation explique la quasiabsence de dispositifs institutionnels publics en Suisse.

L'adoption, en 2006, d'une nouvelle disposition constitutionnelle, accorde désormais à la Confédération des compétences législatives élargies en matière de FPC. II est cependant prématuré d'affirmer que les nouvelles prérogatives se traduiront par une politique de promotion de la FPC en Suisse et par des mesures tangibles d'encouragement.

FPC en entreprises. Nous avons élaboré notre projet à partir des hypothèses de la théorie du capital humain (Becker, 1964). Dans cette approche, l'analyse des dépenses de formation professionnelle des entreprises a donné lieu à de nombreux travaux ces dernières années (pour un état des lieux, $c f$. Barrett et Hövels, 1998). Toutefois, en Suisse, la recherche économique sur la FPC en entreprise est encore peu développée.

Notre projet de recherche (voir encadré 2) ne s'est pas limité à collecter des données sur les coûts assumés par les firmes, mais a aussi permis de 
connaître les bénéficiaires de la FPC, le genre d'activité prioritairement soutenue, les avantages attendus par les entreprises et les modalités d'évaluation des effets.

\section{Encadré 2}

\section{Le projet de recherche « La formation professionnelle continue dans les entreprises publiques et privées en Suisse : coûts, financement et avantage »}

Le projet, doté de $198700 €\left({ }^{*}\right)$, a été mené de 2000 à 2003 par une équipe de chercheurs de l'université de Genève et par un collaborateur d'un institut privé de Bâle. La recherche avait pour objectifs d'estimer les dépenses de formation professionnelle continue (FPC) des entreprises en Suisse, de déterminer la manière dont les ressources sont utilisées et les mécanismes d'évaluation des effets de la FPC. II s'agissait aussi de mettre en évidence les motivations des entreprises à investir ou non dans la FPC. Dans un premier temps, 23 études de cas exploratoires ont été réalisées dans des entreprises privées (17 entreprises) et publiques (6), en Suisse romande (12) et alémanique (1 1). Dans chaque étude de cas, les entretiens ont été menés auprès d'un responsable de formation et de deux collaborateurs. II s'agissait de pré-tester un questionnaire auprès des responsables de formation et de cerner la perception de la FPC auprès des salariés, les raisons pour lesquelles ces derniers se sont ou ne se sont pas formés récemment, et les avantages et les inconvénients de la FPC selon la perception des salariés.

Dans une seconde étape, un questionnaire a été adressé à 12395 entreprises publiques et privées selon un échantillon extrait du Registre fédéral des entreprises. L'échantillon a été stratifié selon la taille (micro-entreprises, petites, moyennes et grandes entreprises), la région linguistique (Suisse alémanique, Suisse romande et Suisse italienne), le statut privé et public, et enfin le secteur d'activité économique (treize catégories de la nomenclature générale des activités économiques * ${ }^{*}$ ). L'échantillon était représentatif de l'ensemble des entreprises exerçant une activité en Suisse. 2157 questionnaires ont été retournés, dont 1758 utilisables (taux de réponse de 14,3\%). La représentativité a été vérifiée par une analyse de la distribution des non-répondants par rapport à l'échantillon d'origine. En raison d'un taux de réponse parfois faible, il a été procédé au regroupement des réponses selon le secteur d'activité économique en sept catégories (primaire ; secondaire ; tertiaire privé ; administration publique, défense, sécurité sociale ; éducation et enseignement; santé et activités sociales; activités foncières et immobilières et autres services). Le questionnaire, inspiré de celui de la $2^{2}$ enquête européenne sur la FPC en entreprise (CVTS2), comportait sept parties (informations générales sur l'entreprise ; programmes et plans de FPC; activités de FPC soutenues; dépenses et recettes de FPC; procédures d'évaluation ; chiffre d'affaires et masse salariale ; raisons de soutenir ou de ne pas soutenir la FPC). Le questionnaire contenait encore deux annexes (statistique du nombre de participants, des heures de FPC par type d'activité ; détail des coûts de formation). Les entreprises dans l'impossibilité de fournir des dépenses effectives ont transmis des estimations; la plausibilité de ces dernières a été systématiquement contrôlée au retour des questionnaires et lors de la saisie des données.

Pour plus d'informations, nous renvoyons le lecteur au rapport de recherche (Hanhart, Schulz, Perez \& Diagne, 2005).

$\left(^{*}\right)$ : toutes les dépenses à l'origine en CHF ont été converties en $€$ au cours moyen de l'année 2001, soit $€ 1$ = CHF 1.51.

$(* *)$ : cette classification est fondée sur celle de l'Union européenne (NACE). Elle permet donc une comparaison avec les statistiques de l'Union européenne. 


\section{LES ENTREPRISES SOUTIENNENT LES SALARIÉS QUALIFIÉS}

Le portrait-type du bénéficiaire de FPC soutenue par l'entreprise, en Suisse, est celui d'un homme, occupant un poste à responsabilité, âgé de 26 à 50 ans. Il participe principalement à des cours externes, à des séminaires et à des conférences dont les thèmes sont étroitement liés à l'activité qu'il exerce. Nous allons affiner ce portrait.

Létude fait ressortir que la participation à la FPC varie selon la position hiérarchique et le niveau de qualification du personnel. Dans près de $40 \%$ des entreprises, une majorité de dirigeants et de cadres supérieurs a bénéficié en 2001 de FPC, alors que ce taux chute à $9 \%$ pour les collaborateurs non qualifiés. En cela, les entreprises suisses ne se différencient pas fondamentalement de leurs homologues européennes (Commission européenne, 2002, p. 32). De manière plus surprenante, dans presque la moitié des micro-entreprises $(<10$ personnes) la majorité des dirigeants a participé à des activités de FPC. Les grandes firmes ( $\geqslant 250$ personnes) semblent adopter un comportement encore plus sélectif, puisque dans seulement $29 \%$ d'entre-elles une majorité de dirigeants et de cadres a bénéficié de FPC ; peut-être les grandes entreprises disposent-elles d'un top management plus qualifié que les établissements de taille inférieure.

Qu'en est-il de l'âge des participants à la FPC ? A priori, les entreprises auraient avantage à favoriser la formation de collaborateurs relativement jeunes, car la période de rentabilisation de l'investissement en formation sera potentiellement plus longue. Les résultats de notre enquête confirment cette hypothèse. Une majorité de participants à des activités de formation continue se recrute parmi les 26 à 50 ans, et ce tant pour les hommes que pour les femmes. Cependant, quel que soit le groupe d'âge considéré, les hommes sont plus nombreux que les femmes (respectivement 65 et $35 \%$ de 26 à 50 ans, et 55 et $45 \%$ pour les personnes de moins de 26 ans) à bénéficier de FPC soutenue par les entreprises, et l'écart augmente avec l'âge. Sans doute ce phénomène reflète-t-il à la fois l'évolution des trajectoires professionnelles et le fait que plus d'hommes se trouvent à des postes à responsabilité. Le taux d'emploi joue aussi un rôle dans le soutien des employeurs, ceux-ci privilégiant les collaborateurs à plein temps. Or, en Suisse, trois emplois à temps partiel sur quatre sont occupés par des femmes (OFS, 2003). Relevons encore que la répartition entre hommes et femmes est assez contrastée selon le secteur d'activité. Dans le secondaire, la participation à des actions de formation continue reflète la distribution des personnes occupées dans l'ensemble des entreprises suisses, à savoir $80 \%$ d'hommes et $20 \%$ de femmes. Dans le tertiaire privé, dans les administrations publiques et dans le secteur de la sécurité sociale, les hommes prédominent dès l'âge de 25 ans. Notons que dans l'enseignement public, la participation féminine est supérieure jusqu'à 50 ans, mais tend à s'égaliser avec l'âge ; en revanche, dans le secteur de la santé, quelle que soit la classe d'âge, les femmes sont largement majoritaires dans un rapport constant de trois participantes pour un participant. On observe une nette différence dans la participation à la FPC lorsque l'on compare globalement les secteurs privé (secondaire et tertiaire confondus) et public : les femmes sont proportionnellement plus nombreuses à accéder à la FPC dans le secteur public ; peut-être observons-nous ici l'un des effets des politiques d'égalité d'accès et de conditions d'emplois mises en œuvre depuis quelques années au sein des administrations publiques. Ces constats mériteraient d'être approfondis en croisant la variable sexe avec d'autres variables telles que le diplôme, la position hiérarchique, la région, mais une telle analyse dépasserait le cadre de cet article.

Dans notre échantillon, moins de $15 \%$ des firmes disposaient de leur propre centre de formation ou appartenaient à un centre de formation interprofessionnel. Notons ici que l'existence d'un centre de formation est étroitement associée à la taille des entreprises. Si peu d'entreprises possèdent leur propre infrastructure de formation, disposent-elles en revanche de plans ou de programmes de formation à l'intention de leurs collaborateurs? Seules $30 \%$ d'entre-elles affirment utiliser de tels instruments pour tout ou partie de leur personnel, mais ce pourcentage croît significativement avec la taille des firmes.

S'agissant des activités de FPC soutenues par les entreprises, les firmes tendent à avantager les séminaires, 
les conférences, les congrès, et la fréquentation de cours externes. D'autres activités, telles que les cercles de qualité, les programmes d'échange entre filiales, les stages hors de l'entreprise, la rotation des personnes sur les postes de travail ( job rotation »), l'autodidactisme pendant les heures de travail (par exemple par la fréquentation d'un centre de documentation) ont été rarement, voire très rarement mentionnées. Nous n'avons pas constaté de grandes différences selon la taille ou le secteur d'activité économique des firmes. En privilégiant la participation à des séminaires, à des conférences et à des congrès, les firmes helvétiques rejoignent leurs homologues autrichiennes, allemandes et des pays nordiques (Nestler et Kailis, 2002, p. 3). Relevons encore une certaine convergence de comportements des entreprises du nord de l'Europe et des firmes helvétiques. Toutefois, les entreprises européennes recourent plus souvent à la rotation des postes de travail et aux cercles de qualité (Nestler et Kailis, 2002, p. 3). Les entreprises helvétiques utilisent peu les stages ${ }^{3}$, à l'instar des entreprises allemandes et autrichiennes (Céreq, 2002, p. 2). Or les trois pays possèdent un système d'apprentissage dual; aussi formulons-nous l'hypothèse que lorsque les firmes accueillent durablement des jeunes en formation professionnelle initiale, elles sont moins enclines par la suite à recevoir ou à soutenir des stagiaires en formation continue.

En Suisse, les firmes soutiennent d'abord les formations associées étroitement à l'exercice du métier, puis les formations en informatique et celles qui sont liées à la gestion et à l'administration des entreprises. Cette observation est valable aussi bien pour les entreprises publiques que privées. L'enquête CVTS2 de 1999 (Commission européenne, 2002, p. 152) montre, à partir d'une répartition des heures par domaine, tous pays confondus, que les formations en informatique arrivent en première position, suivies des processus de formation (« ingénierie et fabrication »), des compétences personnelles et des connaissances du monde du travail. Bien qu'une comparaison stricte des données européennes et suisses s'avère difficile, les résultats donnent à penser que les entreprises helvétiques sont

\footnotetext{
${ }^{3}$ Nous avons distingué dans notre enquête les stages hors de l'entreprise des cours dans un établissement externe. Cette distinction n'existe pas dans l'enquête CVTS.
}

plus restrictives dans leur appui, car elles soutiennent le plus souvent des FPC associées à l'exercice de tâches précises dans la firme.

\section{UNE PARTICIPATION VARIABLE DES ENTREPRISES}

Nous abordons les dépenses consenties par les firmes en faveur de la FPC. Nous avions déjà constaté, lors d'une recherche précédente sur les dépenses de formation professionnelle initiale, un déficit d'information dans les petites et moyennes entreprises (Hanhart \& alii, 1998, p. 62). Cette observation vaut aussi pour les dépenses de FPC, puisque près de la moitié des firmes de notre échantillon ont fourni des évaluations. Celles-ci ont fait l'objet de contrôles de fiabilité.

\section{Plus de la moitié des entreprises financent de la formation continue}

Rappelons qu'en Suisse, les entreprises n'assument généralement pas d'obligation légale en matière de FPC de leur personnel. Aussi est-il intéressant de connaître la proportion des entreprises de notre échantillon ayant financé de la FPC. $61 \%$ des firmes ont déclaré avoir financé de la formation en 2001, alors qu'elles n'étaient que $57 \%$ l'année précédente et $53 \%$ deux ans auparavant. Cette tendance à la hausse se retrouve pratiquement dans tous les secteurs d'activité, ce qui pourrait signifier que la formation professionnelle des salariés est intégrée dans les stratégies de développement d'un nombre croissant d'entreprises en Suisse. Le pourcentage augmente avec la taille : passant de $41 \%$ pour les micro-entreprises à $97 \%$ pour les grandes. D'importantes variations apparaissent aussi par secteur économique. Dans le domaine de la santé, plus de $80 \%$ des institutions ont financé de la FPC, alors que le pourcentage a évolué entre 39 et $46 \%$ dans le secteur tertiaire privé. Nous observons encore des différences entre établissements publics et privés : en 2001 , près de $69 \%$ des administrations et des établissements publics ont financé des activités de FPC au profit de leurs collaborateurs, contre $55 \%$ 
Tableau 1

Dépense moyenne de FPC (Formation professionnelle continue) par entreprise et par collaborateur (en $€$ ) selon le secteur d'activité (calculs fondés sur les équivalents plein-temps) (2001)

\begin{tabular}{|l|c|c|c|}
\hline \multicolumn{1}{|c|}{ Secteur économique } & $\begin{array}{c}\text { Dépense moyenne } \\
\text { par collaborateur }\end{array}$ & $\begin{array}{c}\text { Nombre moyen de } \\
\text { collaborateurs } \\
\text { par entreprise }\end{array}$ & $\begin{array}{c}\text { Dépense moyenne } \\
\text { par entreprise }\end{array}$ \\
\hline Secteur primaire & 705 & 12 & 8108 \\
\hline Secteur secondaire & 491 & 135 & 65843 \\
\hline Secteur tertiaire privé & 655 & 139 & 91176 \\
\hline $\begin{array}{l}\text { Administration publique, défense, sécurité } \\
\text { sociale }\end{array}$ & 438 & 279 & 122071 \\
\hline Éducation et enseignement & 1471 & 56 & 81641 \\
\hline Santé et activités sociales & 662 & 156 & 103073 \\
\hline Total & 831 & 133 & 110606 \\
\hline
\end{tabular}

Source : Hanhart, Schulz, Perez \& Diagne, 2005, p. 46.

des entreprises privées. L'OCDE (2001b) souligne que si la formation continue est un concept largement reconnu, les dispositifs institutionnels publics sont assez peu nombreux dans certains pays membres de cette organisation. En l'occurrence, la quasi-absence de tels dispositifs en Suisse affecte plus les salariés du secteur privé que leurs collègues des administrations et établissements publics.

\section{La dépense varie fortement selon le secteur économique}

La dépense moyenne par entreprise et par collaborateur, en 2001, est très variable selon le secteur d'activité. Elle est en comparaison quatre fois plus élevée dans le secteur de l'éducation et de l'enseignement que dans les administrations publiques; dans les autres secteurs, la dépense moyenne se situe dans une fourchette de 500 à $650 €$. La dépense moyenne par entreprise est très variable selon le secteur, et cette variabilité est le reflet de tailles d'entreprises très différentes.

Il n'est guère possible de comparer systématiquement les dépenses des entreprises suisses à celles de leurs homologues de l'Union européenne pour chaque secteur. En premier lieu, l'enquête CVTS2 n'a pas pris en compte les administrations publiques, les domaines de l'enseignement et de l'éducation, de la santé et des activités sociales. Ensuite, le nombre d'entreprises ayant participé à l'enquête suisse s'est avéré trop restreint pour permettre une désagrégation des résultats identique à celle de l'étude européenne. Enfin, l'enquête CVTS2 a adopté comme unité de calcul le nombre de collaborateurs, indépendamment de leur taux d'activité, alors que nous avons opté pour des équivalents plein-temps. Néanmoins, nous avons été en mesure de situer quelques données suisses dans le contexte européen.

Les dépenses de FPC des entreprises européennes sont très variables d'un secteur économique à l'autre et à l'intérieur d'un même secteur (Commission européenne, 2002, p. 100). Si nous considérons dans le secteur secondaire les deux domaines de l'enquête européenne présentant la plus petite dépense (textile et habillement, respectivement $296 €$ et $451 €$ ) et la plus grande dépense (électricité, gaz et eau, $1413 €$ ), la dépense suisse (742 € en moyenne) dans l'ensemble du secteur secondaire ne semble pas s'écarter des pratiques européennes (Hanhart, Schulz, Perez \& Diagne, 2005, p. 47). En ce qui concerne le tertiaire privé dans l'Union européenne, l'écart entre les deux branches économiques extrêmes est très important : 
Tableau 2

Dépense moyenne de FPC (formation professionnelle continue) en euros par entreprise et par collaborateur selon la taille de la firme

(calculs fondés sur les équivalents plein-temps) (2001)

\begin{tabular}{|l|c|c|c|}
\hline \multicolumn{1}{|c|}{ Taille de l'entreprise } & $\begin{array}{c}\text { Dépense moyenne } \\
\text { par collaborateur } \\
\text { en CHF }\end{array}$ & $\begin{array}{c}\text { Nombre moyen } \\
\text { de collaborateurs } \\
\text { par entreprise }\end{array}$ & $\begin{array}{c}\text { Dépense moyenne } \\
\text { par entreprise } \\
\text { en CHF }\end{array}$ \\
\hline Micro (< 10 pers.) & 845 & 4 & 3211 \\
\hline Petite (10-49 pers.) & 555 & 29 & 11045 \\
\hline Moyenne (50-249 pers.) & 558 & 96 & 53401 \\
\hline Grande ( $\geqslant 250$ pers.) & 919 & 378 & 347658 \\
\hline Total & 831 & 133 & 110606 \\
\hline
\end{tabular}

Source : Hanhart, Schulz, Perez \& Diagne, 2005, p. 48.

$222 €$ pour l'hôtellerie et la restauration et $1004 €$ pour les télécommunications. La dépense des entreprises suisses du tertiaire privé ( $655 €$ ) ne semble pas s'écarter non plus des pratiques européennes. Ces comparaisons amènent à poser une hypothèse : en dépit de l'absence d'une contrainte légale et malgré un subventionnement public très limité, les entreprises suisses semblent investir en moyenne autant que leurs homologues européennes dans la FPC. Nous verrons par la suite que cette appréciation devra être nuancée.

\section{La dépense des micro-entreprises égale presque celle des grandes!}

Comment évolue la dépense moyenne par collaborateur selon la taille des entreprises?

Les dépenses moyennes par collaborateur des petites et moyennes entreprises diffèrent peu. Il en va de même des dépenses des micro et des grandes entreprises ; ce dernier résultat est a priori inattendu et sera relativisé par la prise en compte de la masse salariale. Une analyse statistique fournit une première explication; la distribution des dépenses des micro-entreprises présente une très grande dispersion à la moyenne : au sein des 236 microfirmes de notre échantillon, nous en avons relevé cinq dont la dépense de FPC par collaborateur, en 2001, est égale ou supérieure à $6600 €$, et parmi ces entreprises, quatre relèvent de professions dites libé- rales (secteur médical et paramédical, activités de conseil aux entreprises). Ce constat reflète peut-être un changement dans la nature des activités des micro-entreprises. Avec la tertiarisation marquée de l'économie suisse, les micro-entreprises ne sont plus essentiellement composées d'artisans et de petits industriels, mais également de personnes proposant des services divers, et dont les besoins (voire les obligations légales dans le domaine médical ou fiduciaire par exemple) en FPC augmentent.

Les dépenses des petites et grandes entreprises helvétiques se situent au-dessus de la moyenne des quinze pays de l'Union européenne. Cependant, l'écart n'est sans doute pas aussi important qu'il y paraît pour deux raisons déjà évoquées précédemment: d'une part, nous avons calculé une dépense par équivalent plein-temps alors que les moyennes européennes sont établies sur la base du nombre total de personnes engagées. D'autre part, l'enquête européenne excluait trois domaines d'activité, dont l'éducation et l'enseignement. Or comme nous l'avons constaté précédemment (tableau 1), il s'agit du secteur d'activité dans lequel la dépense de FPC est comparativement élevée en Suisse. En dépit de ces légers biais, nous observons que les petites et grandes entreprises helvétiques investissent en moyenne respectivement $840 €$ et $1390 €$, soit autant que leurs homologues européennes, en tenant compte du niveau élevé des coûts de formation en Suisse (Hanhart, Schulz, Perez $\&$ Diagne, 2005, p. 49). Pour ce qui est des entreprises 
Dépense moyenne de FPC (formation professionnelle continue) par rapport au coût du travail selon le secteur d'activité (2001)

\begin{tabular}{|l|c|}
\hline \multicolumn{1}{|c|}{ Secteur économique } & $\begin{array}{c}\text { Dépense de FPC * } 100 \\
\text { Coût du travail }\end{array}$ \\
\hline Secteur primaire & 1.6 \\
\hline Secteur secondaire & 1.1 \\
\hline Secteur tertiaire privé & 1.3 \\
\hline Administration publique, défense, sécurité sociale & 0.8 \\
\hline Éducation et enseignement & 0.7 \\
\hline Santé et activités sociales & 0.6 \\
\hline Total (tous secteurs confondus) & $\mathbf{1 . 0}$ \\
\hline
\end{tabular}

Source : Hanhart, Schulz, Perez \& Diagne, 2005, p. 51.

de taille moyenne, les firmes helvétiques investissent $840 €$, c'est-à-dire moins que leurs homologues européennes qui dépensent $1000 €$ (Commission européenne, 2002, p. 100). Des études complémentaires seraient nécessaires pour mieux comprendre le relatif sous-investissement en FPC des entreprises de taille moyenne en Suisse par rapport aux firmes européennes.

Soulignons encore une différence selon la région linguistique : les firmes alémaniques ont tendance à dépenser plus pour la FPC de leurs collaborateurs (982 € par personne) que les entreprises romandes (562 € par collaborateur). Peut-être cette observation est-elle due au fait que la FPC occupe aujourd'hui une place plus centrale dans les stratégies de développement des entreprises en Suisse alémanique qu'en Suisse romande.

\section{Un effort de formation inférieur à celui des pays européens}

L'effort consenti en faveur de la FPC peut être estimé par un indicateur mettant en relation les dépenses de FPC et les coûts totaux de personnel (salaires, contributions de l'employeur aux assurances sociales et à la prévoyance professionnelle, frais de formation).

Nous observons ici une situation contrastée selon les secteurs d'activité; les institutions d'éducation et d'enseignement, dont la dépense de FPC par personne est parmi les plus importantes (tableau 3), ne consacrent que $0,7 \%$ de leur coût de personnel à la FPC. Dans cette catégorie, il conviendrait sans doute d'affiner l'analyse en distinguant le corps enseignant des personnels administratifs et techniques; probablement une grande partie des dépenses de FPC estelle consentie en faveur des enseignants, alors que les autres collaborateurs administratifs et techniques ont bénéficié d'un soutien financier comparable à celui des salariés répertoriés dans la rubrique « Administration publique, défense et sécurité sociale ». Les écarts entre secteurs s'expliquent sans doute en grande partie par des différences de masses salariales, qui reflètent aussi de grandes variations dans les niveaux de rémunération selon les domaines d'activité. Nous avons relevé précédemment que les entreprises suisses du secteur secondaire et du tertiaire privé se situaient à un niveau moyen par rapport à leurs homologues européennes. L'indicateur d'effort conduit à nuancer l'appréciation initiale. Ici, les entreprises helvétiques du secondaire et du tertiaire privé consentent un effort moindre que les firmes européennes (Commission européenne, 2002, p. 94). Comment expliquer cette apparente contradiction dans l'interprétation de l'effort des firmes suisses ? D'abord, le niveau moyen plus élevé des salaires helvétiques, comparé aux rémunérations dans les pays de l'Union européenne, influe sur le ratio susmentionné, et explique en partie un indice d'effort bas en Suisse. Ensuite, ce résultat est aussi révélateur 
d'une structure spécifique des coûts de personnel en Suisse, dans laquelle le poids des dépenses de formation est nettement moins important.

L'analyse selon la taille des entreprises indique que les micro-entreprises ont consacré $0,1 \%$ de leur coût de personnel aux dépenses de FPC, le pourcentage étant de $0,3 \%$ pour les petites entreprises, de $0,7 \%$ pour les moyennes et de $1,4 \%$ pour les grandes. À titre de comparaison, les petites firmes européennes affectent entre 1,4 et $1,5 \%$ de leur coût de personnel à la FPC, les entreprises moyennes $2,4 \%$ et les grandes entre 2,3 et 2,7 \% (Commission européenne, 2002 , p. 94). Ces données confirment l'observation tirée de l'étude comparative selon le secteur d'activité économique, à savoir que l'effort de formation consenti par les firmes helvétiques est inférieur à celui de leurs homologues européennes, et ce quelle que soit la taille considérée.

Comment les entreprises financent-elles la FPC ? Il ressort de notre enquête que moins de $20 \%$ des entreprises ont bénéficié d'apports externes (subventions publiques ou privées, émoluments payés par les participants). Le financement par les firmes demeure donc la règle en Suisse ; le constat ne surprend guère dans un pays dépourvu de dispositifs publics de subventionnement en faveur de la formation continue en entreprise, à de rares exceptions près ${ }^{4}$.

En outre, il convient de s'interroger : l'effort relativement réduit des entreprises helvétiques pour la formation de leurs collaborateurs est-il compensé par un investissement personnel accru des salariés? L'enquête internationale sur l'alphabétisation des adultes («International Adult Literacy Survey»), menée de 1994 à 1998, révèle un taux de participation des personnes en emploi à des formations à des fins professionnelles de $31,8 \%$; la Suisse se situe

\footnotetext{
${ }^{4}$ La loi sur la formation continue des adultes du canton de Genève constitue l'une de ces exceptions. Elle prévoit que les autorités cantonales peuvent verser une subvention extraordinaire au Fonds cantonal en faveur de la formation et du perfectionnement professionnel, lorsque le taux de chômage atteint $4 \%$. Ledit Fonds pourra, entres autres, utiliser cette subvention extraordinaire pour le financement d'actions de formation continue organisées par les entreprises privées en faveur de leurs personnel (art. 14 al. $2 \mathrm{a}$ du règlement d'application de la loi sur la formation continue des adultes). Le lecteur notera que cette disposition légale a effectivement permis de soutenir financièrement des actions de formation continue au sein d'entreprises genevoises.
}

ainsi au $10^{\mathrm{e}}$ rang parmi dix-huit nations (OCDE, 2001a, p. 7). L'investissement relativement modeste des entreprises helvétiques n'est donc guère contrebalancé par un effort personnel des salariés. Ce constat devrait susciter une réflexion dans les milieux politiques et économiques, et l'adoption de mesures incitatives, car un sous-investissement en FPC risque à terme d'affecter la compétitivité des firmes et l'attractivité de la place économique suisse.

\section{ÉVALUER LA FORMATION À L'AUNE DE LA SATISFACTION DES SALARIÉS}

Une meilleure connaissance des investissements en formation des entreprises ne saurait se limiter à une analyse des ressources mobilisées et des modalités de financement des dépenses. L'entreprise vise, à travers l'effort de formation, à développer sa capacité productive. Elle est en droit d'en attendre des retombées positives.

L'enquête indique qu'en moyenne, tous secteurs d'activité confondus, $60 \%$ des entreprises disposaient d'une ou de plusieurs procédures formelles d'évaluation. Dans les secteurs secondaire, tertiaire privé et dans le domaine de la santé et des activités sociales, la proportion des entreprises qui appliquent des procédures formelles d'évaluation des effets de la formation est supérieure à la moyenne. En revanche, dans l'enseignement et l'éducation et dans les administrations publiques, l'évaluation formelle des effets de la formation est moins courante. Plus que l'appartenance à un secteur d'activité, la taille des entreprises est déterminante dans la présence ou l'absence de dispositifs formels d'évaluation de la FPC : parmi les firmes répondantes, seule la moitié des microentreprises, mais entre 60 et $70 \%$ des petites et moyennes et presque $80 \%$ des grandes entreprises disposent de tels dispositifs. Sans doute ce constat s'explique-t-il par le fait que, dans les plus petites entreprises, on recourt beaucoup plus à des évaluations informelles (comptes-rendus oraux, observation directe et globale du transfert des nouvelles qualifications et compétences sur le lieu de travail, ...); par ailleurs, ces mêmes petites firmes manquent sans 
doute d'expertises en matière d'évaluation. Observons encore que les entreprises privées évaluent davantage la FPC ( $65 \%$ des firmes) que les administrations et établissements publics (54\%).

En référence au modèle de Kirkpatrick \& Kirkpatrick (2006), l'évaluation porte le plus souvent sur la mesure de la satisfaction des collaborateurs après une activité de FPC, suivie de la vérification de l'application des nouvelles compétences sur le lieu de travail, alors que la validation formelle et la certification des compétences acquises ou le recours à des tests de contrôle sont très rarement cités. L'évaluation des effets de la FPC a généralement lieu immédiatement après l'action ou le programme de formation, l'évaluation dans les mois ou dans l'année qui suit étant beaucoup moins pratiquée. Il apparaît donc que les entreprises évaluent le plus souvent le degré de satisfaction (réactions des participants), et dans une moindre mesure l'efficacité de l'apprentissage (atteinte des objectifs à l'issue de la formation) et la modification des comportements sur le lieu de travail (transfert des nouvelles connaissances). Lévaluation des effets de la formation sur le rendement de l'entreprise (résultats) n'est guère pratiquée; ce dernier constat est corroboré par le fait que l'évaluation n'a presque jamais lieu au-delà de l'année suivant la formation.

$40 \%$ des entreprises ne procèdent pas à des évaluations formelles; elles invoquent d'abord la difficulté à obtenir des résultats fiables (36\% des citations), puis le fait que l'évaluation n'est pas prioritaire à leurs yeux ; ce dernier argument est plus fréquemment mentionné dans les micro-entreprises et au sein des administrations et établissements publics. Enfin, les firmes considèrent qu'une évaluation prendrait trop de temps. Relevons que ni le coût, ni une éventuelle réticence des employés ne constitue un obstacle majeur à l'évaluation.

\section{ACCROÎTRE L'EFFICACITÉ IMMÉDIATE DES SALARIÉS}

Notre échantillon comportait des entreprises soutenant ou non la FPC de leurs employés. Globalement, deux tiers des entreprises ont mentionné des arguments favorables à un financement de la FPC, mais la proportion varie fortement selon la région linguistique : si $80 \%$ des firmes suisses alémaniques sont convaincues de la nécessité de contribuer à la FPC de leurs collaborateurs, ce taux chute à $50 \%$ en Suisse romande et même à $30 \%$ en Suisse italienne. Ceci peut expliquer pourquoi les entreprises alémaniques dépensent plus par collaborateur que leurs homologues romandes, comme évoqué précédemment.

Nous avons interrogé les participants à l'étude sur les raisons qui les incitent à soutenir la FPC de leurs salariés. Nous avons déjà relevé que les entreprises avaient tendance à privilégier les activités de FPC directement liées au poste de travail ; logiquement, les arguments associés aux compétences directement utilisables ont été le plus souvent cités : souhait d'une efficacité accrue des collaborateurs, volonté de maintenir le niveau de compétence, désir d'améliorer la flexibilité des employés. En revanche, les arguments relevant de l'image de l'entreprise, d'éventuelles économies par rapport à l'engagement de nouveaux collaborateurs, de fidélisation à la firme n'apparaissent qu'en fin de liste des citations. Une étude de l'Institut fédéral pour la formation professionnelle en Allemagne (Bundesinstitut für Berufsbildung), citée par Cordier (2001, p. 173), a montré que les entreprises insistent sur l'augmentation du potentiel d'innovation qui sous-tend des efforts de FPC. Cette préoccupation semble également se manifester parmi les entreprises privées en Suisse, mais Cordier relève qu'en Allemagne la FPC a non seulement pour finalité de faciliter l'adaptation des collaborateurs aux changements de l'appareil productif, mais aussi de contribuer à leur développement personnel et à une modification de leur statut, si l'occasion s'en présente. En Suisse, ces deux dimensions supplémentaires ne semblent pas retenir l'attention des entreprises.

Globalement, un tiers des firmes de notre échantillon ne finance pas la FPC de leurs collaborateurs. Le plus souvent, elles estiment que leurs collaborateurs disposent de compétences suffisantes, c'est-àdire que d'éventuels nouveaux problèmes pourront être résolus en interne. En seconde position, les entreprises jugent que la FPC n'est pas nécessaire dans leur domaine d'activité ; l'importance de ces 
deux motifs décroît lorsque la taille des firmes augmente.

Les entreprises doivent aujourd'hui s'adapter rapidement aux changements technologiques et de marchés. Comment envisagent-elles, à terme, de satisfaire leurs besoins en personnel qualifié ? Différentes stratégies sont envisagées : recruter du personnel hors firme, déjà formé ou à former, ou proposer de la FPC aux collaborateurs en place. Notre recherche révèle qu'une stratégie prédomine, quelle que soit la taille des firmes : la formation de nouveaux collaborateurs est mentionnée comme très importante ou importante par près de $90 \%$ des firmes. La FPC du personnel en place arrive en deuxième position : $65 \%$ des grandes entreprises la considèrent comme très importante ou importante dans la qualification du personnel, mais le pourcentage diminue avec la taille pour atteindre $41 \%$ dans les très petites firmes. La troisième stratégie - le recrutement de nouveaux employés déjà formés - est admise comme très importante ou importante par un peu plus de $60 \%$ des entreprises toutes tailles confondues. Le fait que les entreprises tendent à privilégier la formation de nouveaux collaborateurs et le perfectionnement de leurs salariés corrobore une observation que nous avions relevée dans les entreprises formatrices d'apprentis lors d'une recherche antérieure. «Les entreprises formatrices d'apprentis présentent une particularité : elles accordent pratiquement autant d'importance à la formation de nouveaux apprentis qu'à l'offre de cours de perfectionnement professionnel à leurs collaborateurs. Notre enquête tendrait à esquisser les contours d'une véritable stratégie de création et de maintien d'un capital humain, censée répondre durablement aux besoins spécifiques des firmes ...» (Hanhart \& alii, 1998, p. 123).

Ainsi, de nombreuses firmes déclarent que la FPC est un moyen important, voire très important, de qualification de leur personnel. Nous avons confronté cette perception avec les pratiques des entreprises. Nous avons constaté précédemment que l'effort de FPC des entreprises en Suisse, comparé à leurs homologues de l'Union européenne, était en moyenne inférieur à celui des firmes de l'Union européenne. Sans vouloir questionner l'importance accordée à la FPC par les firmes ayant participé à notre étude, force est de constater que l'objectif assigné ici à la FPC (assurer au moins partiellement les besoins à terme de qualifications) est ambitieux, alors que les moyens mis en œuvre semblent plutôt modestes.

Les résultats présentés dans cette partie de l'article se prêtent mal à une comparaison internationale ; cependant, l'enquête européenne sur la formation professionnelle continue (CVTS2) a traité des raisons pour lesquelles, en 1999, les entreprises non formatrices de l'Union européenne se sont abstenues de former. Les firmes avaient la possibilité de choisir au maximum trois raisons, parmi lesquelles le fait d'avoir recruté des personnes possédant les qualifications requises. Cet argument s'apparente à notre proposition libellée « recruter de nouveaux employés ». L'argument du recrutement externe a été prioritairement évoqué par les entreprises non formatrices danoises. Il apparaît, dans une moindre mesure, en Belgique, aux Pays-Bas, en Suède et au RoyaumeUni, alors que les firmes autrichiennes ne l'ont que très rarement invoqué (Nestler \& Kailis, 2002b, pp. 48-49). Il semblerait donc que les entreprises en Suisse tendent moins souvent que leurs homologues européennes à recruter de nouveaux salariés extramuros pour satisfaire leurs besoins en qualifications.

Nous avons mentionné en introduction que la Confédération helvétique disposait, depuis 2006, d'une base constitutionnelle lui permettant de mener une politique active en matière de formation continue. L'un des constats de notre recherche plaide fortement pour l'élaboration et la mise en œuvre, par les pouvoirs publics et à brève échéance, d'une telle politique. Les entreprises tendent à privilégier la formation professionnelle continue de leurs dirigeants et cadres supérieurs. Par ailleurs, une enquête menée récemment auprès d'un échantillon de 33000 ménages en Suisse (OFS, 2006) a montré que «En 2005, seulement $17 \%$ des personnes sans formation post-obligatoire ont pris part à des cours de formation continue, alors que ce fut le cas de plus de la moitié (55\%) des personnes ayant achevé une formation tertiaire [enseignement supérieur]. Ces dernières ont ainsi plus de trois fois plus de chance de suivre de tels cours que les personnes n'ayant pas suivi de formation après la scolarité obligatoire [...] Les personnes disposant d'un diplôme du degré secondaire II [en France, deuxième cycle de l'enseignement secondaire] suivent 
nettement plus souvent des cours de formation continue que celles sans formation post-obligatoire, mais plus rarement que les personnes diplômées $d u$ tertiaire ...»( p. 23). Les informations recueillies auprès des ménages semblent indiquer que l'investissement sélectif - et relativement faible des entreprises suisses en référence au ratio dépenses de FPC/masse salariale - n'est pas compensé par un effort personnel des salariés. À terme, ce constat pourrait sérieusement hypothéquer la compétitivité des entreprises helvétiques. Il pourrait aussi engendrer une segmentation du marché du travail, avec d'une part des emplois occupés par des individus bénéficiant, à leur entrée sur le marché, d'une formation élevée qui se bonifiera par la suite avec l'appui des firmes ; d'autre part, des personnes peu ou pas qualifiées qui ne pourront accéder qu'à des emplois faiblement rémunérés et précaires. Ces mêmes personnes auront peu de chances de se qualifier professionnellement en cours d'emploi, étant donné le risque de chômage et la primauté accordée par les entreprises à la formation des salariés qualifiées.

Dans l'hypothèse où les Chambres fédérales (législatif) décideraient d'une telle politique, il conviendrait à la fois de tenir compte des dispositions déjà prises par les cantons et des investissements consentis aujourd'hui par les salariés (en particulier par les titulaires de diplômes de l'enseignement secondaire II et tertiaire) et les employeurs. D'éventuelles mesures financières d'encouragement de la FPC s'avéreraient efficaces à condition qu'elles induisent une augmentation du volume d'investissement en formation. Cet accroissement n'est pas toujours garanti ; il peut en effet se produire un effet de substitution, l'entreprise ne modifiant pas son comportement à l'égard de la FPC, malgré l'apport de fonds publics. Les firmes utiliseraient les subventions pour couvrir une partie des coûts de formation, sans pour autant générer de nouvelles demandes de FPC (Wolter \& alii, 2004, pp. 17-18). Notre recherche indique que les pouvoirs publics gagneraient à cibler leurs mesures en faveur des salariés faiblement qualifiés afin de leur faire acquérir ou développer des savoirs-faire professionnels non exclusivement utilisables dans un poste de travail précis. Il s'agira aussi de stimuler prioritairement la FPC dans des secteurs d'activité économique où l'investissement privé est relativement faible, tels que le commerce, les transports, l'hôtellerie et la restauration ou encore la construction (OFS, 2001, p. 73).

Une politique de formation continue ne saurait reposer exclusivement sur des mesures visant à encourager l'investissement en formation des entreprises. Cependant, une politique de formation qui sous-estimerait l'apport des firmes à la qualification de leurs collaborateurs perdrait de son efficacité et risquerait même d'être vouée à l'échec. Cette reconnaissance par les pouvoirs publics du rôle joué par les entreprises dans la qualification professionnelle des adultes a un corollaire : que les firmes considèrent leurs dépenses de FPC comme de véritables investissements et qu'elles soient capables d'en rendre visibles les dépenses et les avantages. Notre recherche a montré que cette condition n'est pas souvent remplie aujourd'hui.

\section{Bibliographie}

Barrett A. \& Hövels B. (1998), «Vers un taux de rentabilité de la formation: évaluation de la recherche sur les bénéfices de la formation dispensée par les employeurs », CEDEFOP, Formation professionnelle. Revue européenne, 14, p. 30-39.
Becker G.S. (1964), Human Capital. A Theoritical and Empirical Analysis with Special Relation to Education, Columbia University Press, New York.

Céreq (2002), «L'Europe de la formation tout au long de la vie reste à construire », Céreq-Bref, 187. 
Commission européenne (1999), Formation continue en entreprise : faits et chiffres. Rapport sur les résultats de l'enquête sur la formation continue en entreprise dans les États membres de l'Union européenne en 1994, Office des publications officielles des Communautés européennes, Luxembourg.

Commission européenne (2002), Statistiques sociales européennes. Enquête sur la formation professionnelle continue (CVTS2). Données 1999, Eurostat, Luxembourg.

Cordier B. (2001), « Pratique de l'évaluation de la formation professionnelle continue » in Van Haecht A. (sous la dir. de), Evvaluation et comparaison des politiques et systèmes de formation professionnelle continue en Europe, ULB, Revue de l'Institut de sociologie $n^{\circ}$ spécial, Bruxelles.

Hanhart S., Schulz H.-R. (avec la coll. de Bossio S.) (1998), La formation des apprentis en Suisse. Coûts et financement, Delachaux \& Niestlé, Lausanne.

Hanhart S., Schulz H.-R., Perez S. \& Diagne D. (2005), La formation professionnelle continue dans les entreprises publiques et privées en Suisse : coûts, avantages et financement, Georg Editeur, Genève.

Kirkpatrick D. L. \& Kirkpatrick J. D. (2006), Evaluating Training Programs (6e éd.), San Francisco: Berrett-Koehler Publishers, Inc.
Nestler K. \& Kailis E. (2002). « Formation professionnelle continue en entreprise dans l'Union européenne et en Norvège (CVTS2) », Statistiques en bref, Eurostat, Luxembourg.

OCDE (2001a), Examen thématique de l'apprentissage des adultes. Suisse. Note de synthèse, Paris.

OCDE (2001b), Analyse des politiques d'éducation, Paris.

OFS (Office fédéral de la statistique) (2001), $L a$ formation continue en Suisse 2001, Neuchâtel.

OFS (2003), Statistique trimestrielle de l'emploi (STATEM), Neuchâtel.

OFS (2006), Apprentissage tout au long de la vie et formation continue, Neuchâtel.

Schlaefli A. \& Gonon P. (1999), Weiterbildung in der Schweiz. Situation und Perspektiven. EB-Laenderberichte, Frankfurt/M. : Deutsches Institut für Erwachsenenbildung.

Wolter S. C., Denzler S., Evéquoz G., Hanhart S, Nussbaum O., Ragni T., Schlaefli A. \& Weber B. (2004), Vers un financement de la formation continue orienté sur la demande. Aarau: Centre suisse de coordination pour la recherche en éducation, Rapport de tendance CSRE $n^{\circ} 7$. 
Résumé

\section{Les entreprises suisses: \\ un investissement faible et sélectif dans la formation continue}

Siegfried Hanhart

La formation professionnelle continue (FPC) en Suisse relève d'abord de la responsabilité des individus et des entreprises, la Confédération et les cantons agissant subsidiairement. Il est donc essentiel de saisir le comportement des entreprises à l'égard de la FPC de leurs collaborateurs. De 2000 à 2003, nous avons mené, dans le cadre d'un programme national de recherche, une enquête auprès de 1750 entreprises privées et publiques en Suisse sur leurs dépenses de FPC. Nous en présentons et discutons les principaux résultats: le profil des participants et des activités de FPC soutenues par les entreprises, les dépenses consenties par les employeurs, les modalités d'évaluation des effets de la FPC mises en œuvre dans les firmes, et les raisons évoquées par les employeurs pour soutenir ou pas la FPC de leurs collaborateurs. Lorsque cela est possible, nous comparons les résultats helvétiques avec des données européennes. Nous concluons cet article par une réflexion sur la portée des résultats dans la perspective d'une nouvelle politique publique de formation continue en Suisse.

\section{Mots clés}

Formation professionnelle en entreprise, Financement de la formation professionnelle, Suisse Journal of Economic Literature: M. 53 Training ; I 21 Analysis of Education ; L 22 Firm Organization 